\author{
Marquette University \\ e-Publications@Marquette
}

$12-2008$

\title{
Dependence of the Performance of Single Photon Avalanche Diodes on the Multiplication Region Width
}

\author{
David A. Ramirez \\ University of New Mexico \\ Majeed M. Hayat \\ Marquette University, majeed.hayat@marquette.edu \\ Mark A. Itzler \\ Princeton Lightwave, Inc.
}

Follow this and additional works at: https://epublications.marquette.edu/electric_fac

Part of the Computer Engineering Commons, and the Electrical and Computer Engineering Commons

\section{Recommended Citation}

Ramirez, David A.; Hayat, Majeed M.; and Itzler, Mark A., "Dependence of the Performance of Single Photon Avalanche Diodes on the Multiplication Region Width" (2008). Electrical and Computer Engineering Faculty Research and Publications. 591.

https://epublications.marquette.edu/electric_fac/591 
Marquette University

e-Publications@Marquette

\section{Electrical and Computer Engineering Faculty Research and Publications/College of Engineering}

This paper is NOT THE PUBLISHED VERSION; but the author's final, peer-reviewed manuscript. The published version may be accessed by following the link in the citation below.

IEEE Journal of Quantum Electronics, Vol. 44, No. 12 (December 2008): 1188-1195. DOI. This article is (C) Institute of Electrical and Electronic Engineers (IEEE) and permission has been granted for this version to appear in e-Publications@Marquette. Institute of Electrical and Electronic Engineers (IEEE) does not grant permission for this article to be further copied/distributed or hosted elsewhere without the express permission from Institute of Electrical and Electronic Engineers (IEEE).

\section{Dependence of the Performance of Single Photon Avalanche Diodes on the Multiplication Region Width}

David A. Ramirez

Department of Electrical and Computer Engineering, University of New Mexico, Albuquerque, NM Majeed M. Hayat

Department of Electrical and Computer Engineering, University of New Mexico, Albuquerque, NM Mark A. Itzler

Princeton Lightwave, Inc., Cranbury NJ

\section{Abstract:}

The dependence of the performance of separate-absorption-multiplication (SAM) single-photon avalanche diodes (SPADs) on the width of the multiplication region is theoretically investigated. The theory is applied to SAM SPADs with InP homojunction multiplication regions and InAIAs-InP heterojunction multiplication regions. In both cases the absorber layer is InGaAs. Two scenarios for the dark counts are considered: (i) lowtemperature operation, when the number of dark carriers is dominated by field-assisted mechanisms of band- 
to-band tunneling and tunneling through defects; and (ii) room-temperature operation, when the number of dark carriers in the multiplication region is dominated by the generation/recombination mechanism. The analysis utilizes a generalized theory for breakdown probability, which takes into account the random locations where dark and photogenerated carriers are produced in each layer. Depending upon the detector temperature, as the width of the multiplication region is increased the effects from the reduction in the number of dark carriers due to field-assisted generation mechanisms are counteracted by the effects from the elevation in the number of generation/recombination dark carriers. Thus, there exists an optimal width of the multiplication region that achieves the best performance of the SPAD.

\section{Keywords}

Diodes, Tunneling, Detectors, Radiative recombination, Electric breakdown, Avalanche photodiodes, Laser modes, Silicon, III-V semiconductor materials, Avalanche breakdown

\section{SECTION I. Introduction}

Single-Photon avalanche photodiodes (SPADs) are very important devices in applications such as satellite laser ranging [1], deep-space laser communication [2], time-resolved photon counting [3], quantum key distribution [4], quantum imaging [5], and quantum cryptography [6], [7]. While silicon SPADs have already shown very good performance in various applications in the 400-900 $\mathrm{nm}$ range their performance is degraded drastically when they are operated in the range 1.06-1.55 $\mu \mathrm{m}$. For applications in the telecommunication wavelengths range, i.e., 1.3-1.55 $\mu \mathrm{m}$, devices with a narrower bandgap than silicon, mainly III-V compounds, are utilized. Nevertheless, the lack of a comprehensive model that relates the device physical parameters and the device performance has contributed to the challenge in developing high-performance SPADs in the 1.3-1.55 $\mu \mathrm{m}$ range.

The performance of a SPAD is primarily measured by its photon detection efficiency (PDE) and its dark count rate (DCR). The PDE is the product of the detector quantum efficiency and the avalanche breakdown probability. The DCR constitutes false counts and it is a measure of how noisy the detector is. Dark counts originate from dark carriers generated in the absence of illumination; the larger the number of dark carriers, the larger the dark-count probability is [8]. There are several mechanisms that contribute to the concentration of dark carriers. At high electric fields, the dark-carrier concentration is strongly affected by band-to-band tunneling, which depends exponentially on the electric field [9], and it constitutes a limiting factor in the low excess-noise and fast avalanche photodiodes (APDs) that have thin multiplication regions. Another important mechanism that contributes to the number of dark carriers is tunneling through defects. It has been reported that in some materials the tunneling currents due to defects concentration is higher than that of band-to-band tunneling [10].

Besides the strength of the electric field and the properties of the material, the probability of a carrier triggering an avalanche breakdown is determined by the place where it is born [11]. A carrier created in the start of the multiplication region has a greater probability of triggering an avalanche event compared to that created close to the end of the multiplication region. This is because a primary carrier created early on in the multiplication region has a larger distance to travel compared to those created close to the end of the multiplication region. For example, for a separate absorption separate multiplication (SAM) APD, a carrier created in the absorption layer is more likely to cause an avalanche compared to that created in the multiplication region. More generally, the dependence of the breakdown probability on the birth location of a carrier is crucial in determining the SPAD's performance when the number of dark carriers inside the absorption and multiplication layers is taken into account. As there has been a recent interest in optimizing the width of the multiplication layer for the best DCR and PDE performance [10], and since the width of the multiplication layer significantly affects the electric field (and hence tunneling current), it is important to have a model that can predict the DCR and PDE required 
for Geiger-mode operation for various SPAD structures and geometries while taking into account the types of dark carriers and the randomness in the location where they are generated in the absorption and multiplication layers.

The main focus of this paper is to report theoretical results based on new modeling tools that shed light on the dependence of the performance of SAM SPADs on the width of the multiplication region by comparing the effects of field-assisted tunneling with temperature-assisted dark carriers as the width is varied. This study also reveals the characteristic difference in the performance between low-temperature operation and roomtemperature operation while identifying and quantitatively examining the main factors that govern the performance of the SPAD. Moreover, an aspect of importance that had not been explored before, namely, the random locations where carriers are born in each layer, is thoroughly analyzed and studied. In particular, we assume that photogenerated carriers are generated in the absorber at random locations according to an exponential probability density function (pdf). On the other hand, dark carriers are assumed to be generated randomly in the multiplication region and the absorber according to a uniform pdf in each layer. To calculate the generalized breakdown probability for all the carriers generated in the SPAD we use the recursive dead-space multiplication theory (DSMT) according to a field-dependent spatial distribution of carriers [11], [12]. In addition, along with the DCR and PDE, the single-photon quantum efficiency (SPQE) is also used as a figure of merit to assess the SPAD's performance [8]. The ability of the SPQE to admit an optimal operating overbias makes it a very useful metric [12]. The theory developed is applied to SPADs that operate in a short-pulse gated-mode regime, in the 1.3-1.55 $\mu \mathrm{m}$ range, with InP homojunction multiplication regions and InAIAs-InP heterojunction multiplication regions. In both cases the absorber layer is InGaAs.

The remainder of this paper is organized as follows. In Section II, we discuss the theoretical model developed; this section includes a review of the different dark-carrier generation mechanisms considered, the calculation of the generalized breakdown probability, which makes use of the DSMT, and the metrics of performance. Section III is devoted to applying the developed theory to two different device structures: (i) InGaAs/InP homojunction SAM SPAD with InGaAs absorber and InP homojunction multiplication region, and (ii) InAIAs-InP heterojunction multiplication region. The conclusions are presented in Section IV.

\section{SECTION II. Model}

In this section we draw upon existing models for dark current [10], [13], breakdown probability [11], [12] and SPAD-performance metrics [8], [12] to develop new expressions for distributed breakdown probability for SAM SPADs and their performance.

\section{A. Review of Dark Current Model}

The dominant mechanism of dark-carrier generation in a specific SPAD will depend upon its physical structure and operating conditions such as the bias voltage, repetition rate in gated operation, and temperature. In our study we have considered dark-carrier generation in both of the absorption and multiplication regions. In both regions the mechanisms to be considered are GR, band-to-band tunneling and tunneling through defect states. Accordingly, the number of dark carriers generated per second in the absorber is $N_{d, \text { abs }}=N_{\text {gen,abs }}+N_{\text {def,abs }}+$ $N_{\text {tun,abs. }}$ In the same way, the number of dark carriers generated per second in the multiplication region is given by $N_{d, \text { mul }}=N_{\text {tun,mul }}+N_{\text {def,mul }}+N_{\text {gen,mul }}$.

The GR current density, which is the dominant mechanism of dark-carrier generation at low voltage, is given by the expression [13]

$$
J_{\text {gen }}=\frac{q n_{i} W}{\tau_{\text {eff }}}(1-\exp (q V / 2 \mathrm{kT}))
$$


where $W$ is the width of the depletion region, $n_{i}$ is the intrinsic carrier concentration, $V$ is the applied voltage, and $\tau_{\text {eff }}$ is the effective carrier lifetime. (The units of $J_{\text {gen }}$ are Amperes per square meter.) Thus, the number of dark carriers due to GR is $N_{\text {gen }}=J_{\text {gen }} A / q$, where $A$ is the SPAD's cross-sectional area, and $q$ is the charge of the electron.

At high electric fields, the dominant mechanism of dark-carrier generation is tunneling [13]. Consequently, tunneling currents become very important for thin multiplication layers [14]. Generally, tunneling current increases exponentially as the electric field increases [9]; more precisely [13]

$J_{\text {tun }}=\frac{\sqrt{2 m^{*}} q^{3} E_{m} V}{4 \pi^{2} \hbar^{2} E_{g}^{1 / 2}} \exp \left(-\frac{\theta \sqrt{m^{*}} E_{g}^{3 / 2}}{q E_{m} \hbar}\right)(2)$

where $E_{m}$ is the electric field, $V$ is the voltage across the avalanche region, $m^{*}$ is the electron effective mass, and $\theta$ is a parameter that depends on the shape of the tunneling barrier. As in the case of $N_{\text {gen }}$, the number of dark carriers in the avalanche region due to band-to-band tunneling is $N_{\text {tun }}=J_{\text {tun }} A / q$.

Defects in the material also contribute to increase the dark-carrier generation [9], [10], [15]. The tunneling current density due to defect states is given by the expression [10]

$J_{\mathrm{def}}=\frac{A_{d} E_{m} V N_{T} \exp \left(\frac{-\left(B_{1} E_{B 1}^{3 / 2}+B_{2} E_{B 2}^{3 / 2}\right)}{E_{m}}\right)}{N_{v} \exp \left(\frac{-B_{1} E_{B 1}^{3 / 2}}{E_{m}}\right)+N_{c} \exp \left(\frac{-B_{2} E_{B 2}^{3 / 2}}{E_{m}}\right)}$ (3)

where $\left.A_{d}=q^{3} \sqrt{\left(2 m_{r}\right) /\left(E_{g}\right.}\right) /\left(4 \pi^{3} \hbar^{2}\right), m_{r}=\left(2\left(m_{c} m_{l h}\right)\right) /\left(m_{c}+m_{l h}\right)$ is the reduced effective mass, $m_{c}$ being the conduction band effective mass and $m_{\mathrm{lh}}$ being the light hole effective mass, $B_{1}=$ $\pi\left(m_{\mathrm{lh}} / 2\right)^{1 / 2} /(2 q \hbar)$, and $B_{2}=\pi\left(m_{c} / 2\right)^{1 / 2} /(2 q \hbar)$. In the above expression, $\mathrm{E}_{B 1}$ is the barrier height of tunneling from valence band to trap and is equal to $a E_{g}(a<1)$, and $E_{B 2}$ is the barrier height of tunneling from trap to the conduction and is equal to $(1-a) E_{g}$. The quantities $N_{v}$ and $N_{c}$ are the light hole valence and conduction band density of states and $N_{T}$ represents the number of defects per unit volume [10]. The number of dark carriers in the avalanche region due to defects states is $N_{\mathrm{def}}=J_{\mathrm{def}} A / q$. The average number of dark carriers generated in the SPAD is given by

$N_{d}=N_{d, \mathrm{mul}}+N_{d, \mathrm{abs} \cdot(4)}$

\section{B. Calculation of Breakdown Probability}

In order to apply the DSMT to calculate the generalized breakdown probabilities for all the carriers generated in the SPAD illustrated in Fig. 1 [11], [12], we use (i) the nonlocalized ionization coefficients, also called enabled ionization coefficients (the ionization coefficient assumed once the carrier travels the dead-space distance), and the threshold energies for each material [16], and (ii) the electric-field profile through the device. The nonlocalized electron and hole ionization coefficients and threshold energies for $\ln P, \ln G a A s$, and $\ln G a A s P$ are readily available [16]-[17][18]. 

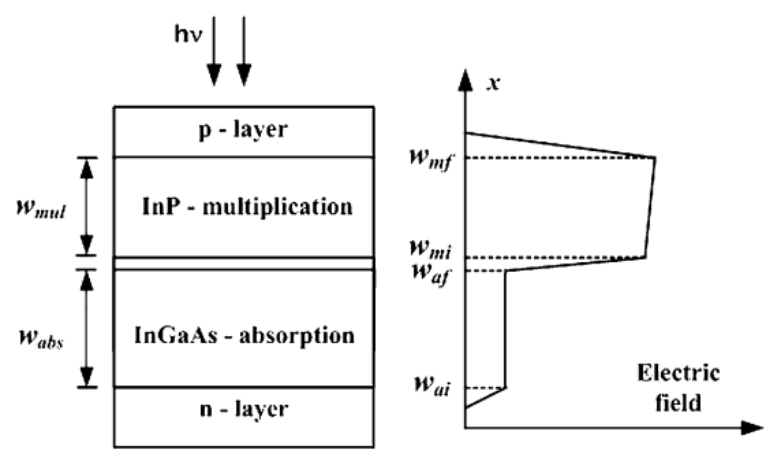

Fig. 1. Device structure and electric-field profile of a SAM SPAD with InP multiplication region and InGaAs absorber.

\section{Probability Density Function of the Free Path}

We have adopted the following shifted-exponential model for the probability densities of the distance to ionization, $y$, measured from the location, $x$, where a carrier is born [11]. For an electron born at location $x$, with $\alpha$ being the enabled ionization coefficient, the probability that it impact ionizes at location $y$ and assuming that electrons move to the direction of increasing $x$ is [19]

$$
\begin{array}{r}
h_{e}(y \mid x)=\alpha(y) \exp \left(-\int_{x+d_{e}(x)}^{y} \alpha(u) d u\right), \\
y \geq x+d_{e}(x)
\end{array}
$$

where $d_{e}(x)$ is the dead space of an electron born at location $x$, and $h_{e}(y \mid x)=0$ when $y<x+d_{e}(x)$. The dead space is the distance a carrier must travel within the SPAD before acquiring the energy threshold needed for effecting an impact ionization; $d_{e}(x)$ satisfies the equation [19]

$E_{\mathrm{th}, e}\left(x+d_{e}(x)\right)=q \int_{x}^{x+d_{e}(x)} E(u) d u(6)$

where $E_{\mathrm{th}, e}(x)$ is the ionization threshold energy for electrons at location $x$ in the SPAD (this energy varies from layer to layer).

There are similar expressions for holes:

$$
\begin{array}{r}
h_{h}(y \mid x)=\beta(y) \exp \left(-\int_{y}^{x-d_{h}(x)} \beta(u) d u\right), \\
y \leq x-d_{h}(x)
\end{array}
$$

where $d_{h}(x)$ is the dead space of a hole born at location $x$, and $h_{h}(y \mid x)=0$ when $y>x-d_{h}(x)$. In the case where the field is constant, the position-independent dead space is calculated using $d=E_{t h} / q E$ [16]. The equations from (5) to (7) of the DSMT are generalized equations; they constitute a powerful tool that allows us to model APDs with any electric-field profile and any structure, like multilayer devices with heterostructure multiplication regions to be reviewed next.

\section{Breakdown Probability}

Suppose that we know the total electron and hole population, $Z(x)$, resulting from a parent electron born at $x$, and the total electron and hole population, $Y(x)$, resulting from a parent hole born at $x$, where $0 \leq x \leq w$, 
and $w$ is the width of the SPAD. We define $P_{Z}(x)$ as the probability that $Z(x)$ is finite, and similarly, $P_{Y}(x)$ as the probability that $Y(x)$ is finite [11]. These quantities reflect the non-breakdown probabilities for carriers generated at location $x$ anywhere in the SPAD. Thus, for example $1-P_{Z}(x)$ is the probability that $Z(x)$ is infinite, which is precisely the case when avalanche breakdown occurs. On the other hand, the probability that an electron-hole pair born at $x$ collectively triggers an avalanche breakdown is $P_{b}(x)=1-P_{Z}(x) P_{Y}(x)$. Recursive integral equations describing $P_{Z}(x)$ and $P_{Y}(x)$ are developed elsewhere [11] and are repeated here for completeness:

$$
\begin{aligned}
P_{Z}(x) & =\int_{w-x}^{\infty} h_{e}(\xi \mid x) d \xi \\
& +\int_{0}^{w-x}\left(P_{Z}^{2}(x+\xi) P_{Y}(x+\xi)\right) h_{e}(\xi \mid x) d \xi \\
P_{Y}(x) & =\int_{x}^{\infty} h_{h}(\xi \mid x) d \xi \\
& +\int_{0}^{x}\left(P_{Y}^{2}(x-\xi) P_{Z}(x-\xi)\right) h_{h}(\xi \mid x) d \xi .
\end{aligned}
$$

(8)(9)

These integral equations can be solved using a straightforward numerical iterative approach similar to that described in [11].

Let us assume that the electron-hole pairs are created at random locations in the absorption and multiplication regions extending from $x=w_{a i}$ to $x=w_{m f}$, as shown in Fig. 1. We also assume that holes (electrons) are transported in the positive (negative) $x$ direction. Moreover, let $f(x)$ denote the pdf of the birthplace of the parent electron-hole pair. Thus, the average probability that an electron-hole pair, randomly generated in the interval $\left[w_{a i}, w_{m f}\right]$ according to the pdf $f(x)$, triggering an avalanche breakdown is given by

$$
Q_{f}=\int_{w_{a i}}^{w_{m f}} f(x)\left(1-P_{Z}(x) P_{Y}(x)\right) d x \cdot(10)
$$

The expression for $Q_{f}$ represents the general form of the breakdown probability for any random distribution of carriers and it accounts for avalanche breakdown occurring either in the absorption or multiplication regions. We can further specialize this expression for two distinct forms of $f$ representing the following physical scenarios: (a) the scenario for which the avalanche breakdown is triggered by electron-hole pairs photogenerated inside the absorption region, in which case we denote $f$ by $f_{\mathrm{ph}}$; and (b) the scenario for which the avalanche breakdown is initiated by dark carriers randomly generated in either the absorption or the multiplication region, in which case we denote $f$ by $f_{d}$. The use of $f_{\mathrm{ph}}$ and $f_{d}$ in $\underline{10)}$ will lead to the injectedcarrier breakdown probability, $Q_{\mathrm{ph}}$, and the distributed-carrier breakdown probability, $Q_{d}$, respectively. The former represents the breakdown probability caused by a carrier pair photogenerated in the absorber; on the other hand, the latter represents the breakdown probability caused by a dark carrier that is randomly generated inside the SPAD, taking into account the dark carriers generated in the multiplication and the absorption regions.

Let us consider first the case where the avalanche breakdown is triggered by dark carriers randomly generated in the SPAD. In this case the pdf $f$ is given by, as depicted in Fig. 2, 


$$
\begin{gathered}
f_{d}=\frac{A}{w_{a f}-w_{a i}}\left(u\left(x-w_{a i}\right)-u\left(x-w_{a f}\right)\right) \\
+\frac{B}{w_{m f}-w_{m i}}\left(u\left(x-w_{m i}\right)-u\left(x-w_{m f}\right)\right)
\end{gathered}
$$

where $A=\left(N_{d, \text { abs }}\right) /\left(N_{d, \text { abs }}+N_{d, \text { mul }}\right), B=\left(N_{d, \mathrm{mul}}\right) /\left(N_{d, \text { abs }}+N_{d, \text { mul }}\right)$, and $u(x)$ is the unit step function. Note that $A$ (resp. $B$ ) is the probabilities that an arbitrary dark-carrier pair already generated in the SPAD was actually generated in the absorption (resp. multiplication) region. The quantities $w_{m i}$ and $w_{m f}$ respectively represent the start and end of the multiplication region, and $w_{a i}$ and $w_{a f}$ respectively represent the start and end of the absorption region, where we have assumed $\left(w_{m f}-w_{m i}\right)+\left(w_{a f}-w_{a i}\right) \approx w$ (see Fig. 1). (In all our calculations we have neglected the effect of the charge layer, which is between the absorber and the multiplication region, leading to the approximation $w_{a f} \approx w_{m i}$.) Consequently, the probability $Q_{d}$ simplifies to

$$
\begin{aligned}
Q_{d} & =\frac{N_{d, \mathrm{abs}}}{N_{d, \mathrm{abs}}+N_{d, \mathrm{mul}}} \frac{1}{w_{a f}-w_{a i}} \\
& \times \int_{w_{a i}}^{w_{a f}}\left(1-P_{Z}(x) P_{Y}(x)\right) d x \\
& +\frac{N_{d, \mathrm{mul}}}{N_{d, \mathrm{abs}}+N_{d, \mathrm{mul}}} \frac{1}{w_{m f}-w_{m i}} \\
& \times \int_{w_{m i}}^{w_{m f}}\left(1-P_{Z}(x) P_{Y}(x)\right) d x
\end{aligned}
$$

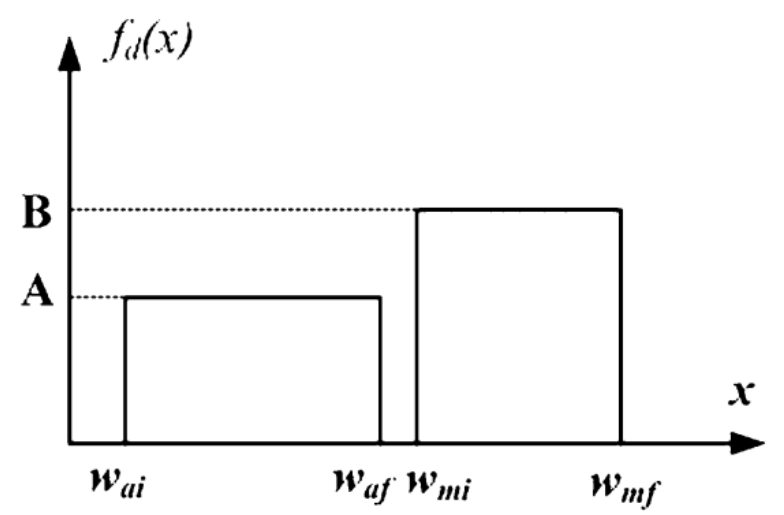

Fig. 2. Probability density function $f_{d}$ for the case where the avalanche breakdown is triggered by dark carriers randomly generated in the SPAD.

In the case of the injected-carrier breakdown probability, the absorption of photons in the absorption region obeys an exponential behavior. Hence, $f_{\mathrm{ph}}$ will be of the form $f_{\mathrm{ph}}(x)=C_{1} e^{-C_{2} x}$, for $w_{a i} \leq x \leq w_{a f}$, and $f_{\mathrm{ph}}=$ 0 elsewhere. For simplicity, we set $x=w_{a i}=0$ and therefore $w_{a f}=w_{\mathrm{abs}}$, which is the width of the absorber (see Fig. 1). The constant $C_{2}$ can be determined by equating $\int_{0}^{w_{a b s}} C_{2} e^{-C_{2} x} d x$ to the SPAD's quantum efficiency, $\eta$. This yields $C_{2}=-\ln (1-\eta) / w_{\mathrm{abs}}$. The constant $C_{1}$, on the other hand, is chosen so that $f_{\mathrm{ph}}$ has unit area, as we would expect from a valid pdf; this yields $C_{1}=C_{2} / \eta$. In summary, the injected-carrier breakdown probability is given by

$$
\begin{aligned}
Q_{\mathrm{ph}}= & \frac{-\ln (1-\eta)}{\eta w_{\mathrm{abs}}} \int_{0}^{w_{\mathrm{abs}}} \exp \left\{\frac{\ln (1-\eta)}{w_{\mathrm{abs}}} x\right\} \\
& \times\left(1-P_{Z}(x) P_{Y}(x)\right) d x
\end{aligned}
$$




\section{SPAD Performance}

The traditional performance metrics, photon-detection efficiency and the dark-count rate are respectively defined as PDE $\triangleq Q_{\mathrm{ph}}$ and DCR $\triangleq N_{d} Q_{d}$. Additionally, the single-photon quantum efficiency is another useful metric to assess the SPAD performance [8], [12]. The latter is defined as the probability that a photon triggers an avalanche breakdown, given that an optical pulse is present and at least one photon impinges on the SPAD, and provided that no dark carrier triggers a breakdown. Mathematically, it is given by

$\mathrm{SPQE}=\frac{\left(1-P_{d}\right) P_{\mathrm{opt}}}{p_{o}}(14)$

where $P_{d}$ is the dark count probability, calculated throughout the absorption and the multiplication regions altogether. The quantity $P_{\text {opt }}$ is the probability that at least one photogenerated carrier in the absorber triggers the avalanche, and $p_{o}$ is the probability that one photon impinges on the SPAD during the detection time. The dark count probability is given by

$P_{d}=1-e^{-N_{d} Q_{d}}$

where $N_{d}$ is the average number of dark carriers generated in the SPAD (calculated in (4)). Note that in Kang et al. [8], the breakdown probability $Q_{\mathrm{ph}}$ is used in place of $Q_{d}$; however, the use of $Q_{d}$, as done here, accounts for dark-carrier generation at random locations across the entire device. The quantity $P_{\text {opt }}$ is calculated using the following expression:

$P_{\mathrm{opt}}=1-e^{-\eta Q_{\mathrm{ph}} N_{o}}$

where $\eta$ is the detector quantum efficiency and $N_{o}$ is the average number of photons per pulse.

\section{SECTION III. Results}

The theory described in the previous section is applied to SAM SPADs with InP homojunction multiplication regions and InAIAs-InP heterojunction multiplication regions. In both cases the absorber layer is InGaAs. In the case where GR dark carriers are included the operating temperature is $300 \mathrm{~K}$. The intrinsic carrier concentration, $n_{i}$, for $\operatorname{InP}$ used in our simulations is $1.2 \times 10^{8} \mathrm{~cm}^{-3}[20]$ while the effective carrier lifetime, $\tau_{\text {eff }}$, in the InP multiplication region is taken as $320 \mathrm{~ns}$ [21]. Moreover, the number of defects per unit volume, $N_{T}$, is chosen as $8 \times 10^{-4}$ according to [10]. On the other hand, the value of the dimensionless parameter, $\theta$, is taken as 1.26, which is the value of $\theta$ for InAlAs according to [22]. This approximation is made due to the lack of precise knowledge of the value of $\theta$ for InP. Finally, the position of the trap is set at $0.75 E_{g}(a=0.75)$ above the valence band [10]. Fig. 1 illustrates the structure and the electric-field profile of the SPAD with InP homojunction multiplication region. A schematic of the electric-field profile across the device is also shown.

To see the role played by the width of the multiplication region on the performance of the SPAD, we have calculated the PDE, DCR and SPQE curves, as the width of the multiplication region is varied, considering two scenarios: (i) low-temperature operation, when the dominant dark-carrier-generation mechanism is fieldassisted and (ii) room-temperature operation, when the dominant mechanism of dark-carrier generation is temperature assisted. The comparison of the performance of the SPAD under these scenarios will illustrate the characteristic difference in the performance between low-temperature operation and room-temperature operation and how this attribute varies as the multiplication-region width is changed. 


\section{A. InGaAs/InP Homojunction SAM Photodiode}

The DCR, PDE, and SPQE for a SAM SPAD with InP homojunction multiplication region of width in the range 500$2000 \mathrm{~nm}$ and absorber of $1 \mu \mathrm{m}$ were calculated. It is expected that the effect on the number of dark carriers, and hence on the DCR, of the temperature-assisted generation of dark carriers is more relevant at lower bias voltages since as we increase the bias voltage the dark-carrier generation will be dominated by field-assisted mechanisms. Fig. 3 shows the calculated DCR as a function of the normalized excess applied voltage for three different widths of the multiplication region. The normalized excess applied voltage is defined as $\Delta V / V_{\mathrm{BR}}$, where $\Delta V=\left(V-V_{\mathrm{BR}}\right), V_{\mathrm{BR}}$ is the breakdown voltage and $V$ is the voltage across the device. The solid lines correspond to the case when field-assisted and temperature-assisted generation of dark carriers are both present in the model. For clarity, we also show the case when only field-assisted generation is taking place (dashed lines). The figure shows that at higher normalized excess bias voltages the DCR curve is almost completely dictated by tunneling effects for all the widths of the multiplication region. It is also noticed that the effect of temperature-assisted dark carrier generation on the DCR is more important in devices with thick multiplication regions, e.g., $>800 \mathrm{~nm}$. On the other hand, for devices with thin multiplication regions the DCR curve is dominated, over almost the whole range of normalized excess voltages, by field-assisted mechanisms.

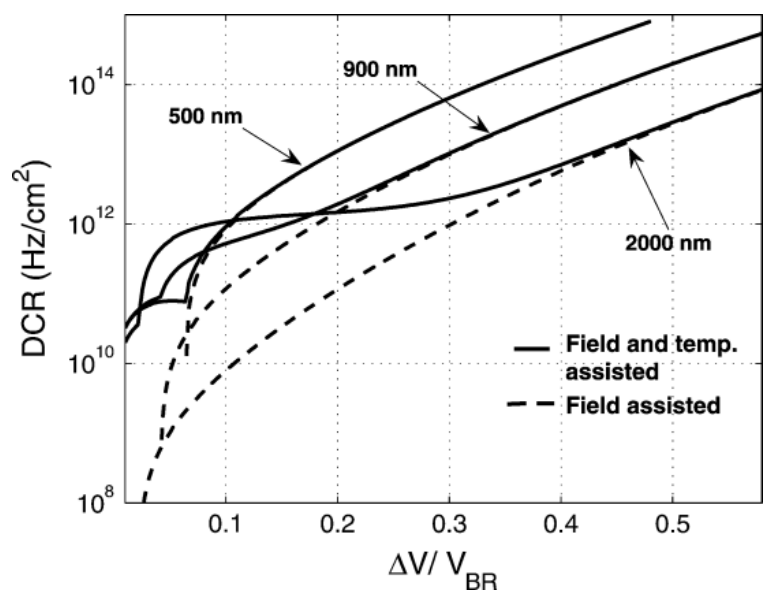

Fig. 3. DCR versus normalized excess voltage for $500 \mathrm{~nm}, 900 \mathrm{~nm}$, and $2000 \mathrm{~nm}$ multiplication region widths. Dashed lines correspond to the case when GR dark carriers are absent and solid lines correspond to the case when both field-assisted and GR dark carriers are present.

Fig. 4 shows the calculated PDE versus DCR for InP multiplication regions of 700, 900, 1200 and $2000 \mathrm{~nm}$, and an InGaAs absorption layer of $1 \mu \mathrm{m}$. There are two groups of curves generated according to the different mechanisms for dark-carrier generation; in the figure these two groups are labeled by their respective ellipses. The lower group of curves corresponds to the cases for which both field-assisted and temperature-assisted generation of dark carriers are included. On the other hand, in the upper group of curves we consider fieldassisted generation of dark carriers only. It can be seen that the PDE versus DCR behavior varies as we include temperature-assisted dark carriers along with field-assisted dark carriers. In the case when only field-assisted dark-carrier generation is considered (upper group), the calculated PDE, for a given DCR, is higher as the multiplication region becomes wider. On the other hand, in the case for which both mechanisms of dark-carrier generation are considered (lower group) we observe two distinct behaviors as the width of the multiplication region increases. First, for the low values of the DCR $\left(<10^{11} \mathrm{~Hz} / \mathrm{cm}^{2}\right)$, we see an improvement in PDE as the width of the multiplication region is increased. However, for larger DCR values, the PDE degrades as the multiplication region becomes wider. Hence, the calculated results illustrated in Fig. 4 suggest that in cooled devices, the performance will improve as we increase the width of the multiplication region. However, for devices working at room temperature the increment in PDE, due to a wider multiplication region, is 
counteracted by an increment in DCR and the performance will be degraded as the multiplication region becomes wider. The improvement in the PDE versus DCR characteristics at low temperatures is attributable to fact that as the width of the multiplication region increases the tunneling current decreases due to the lower electric field. It should be pointed out that the maximum value of the PDE versus DCR curve is determined by the quantum efficiency $\eta$, which in this case is 0.5 .

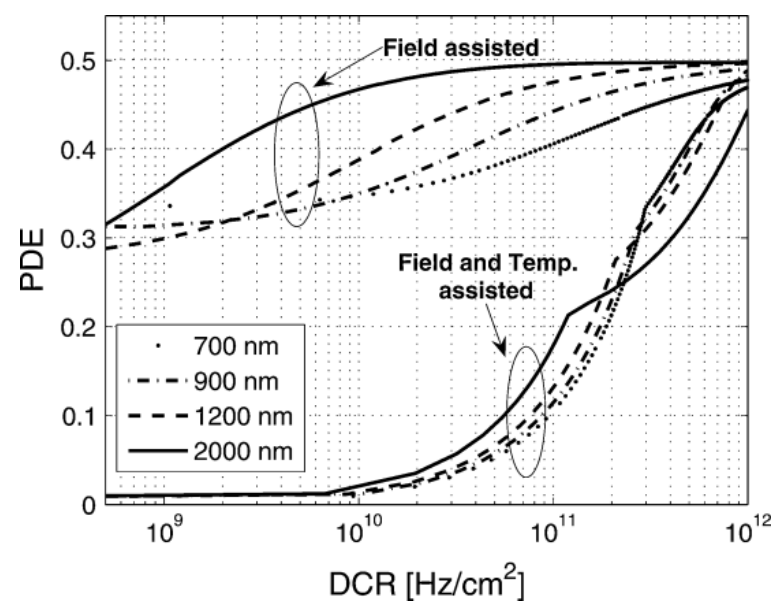

Fig. 4. PDE versus DCR for InP multiplication regions of $700,900,1200$, and $2000 \mathrm{~nm}$. The absorber is a $1 \mu \mathrm{m}$ layer of InGaAs. The maximum value of the PDE versus DCR curve is determined by the quantum efficiency $\eta$, which in this case is 0.5 .

Our calculations of the SPQE, as a function of the applied bias, indicate a similar trend to that suggested by the PDE versus DCR curves. Moreover, the SPQE curves provide further insight by suggesting an optimal thickness of the multiplication region that achieves the highest SPQE at the appropriate applied voltage. Fig. 5 (solid lines) shows that the peak value of each SPQE curve increases as the width of the multiplication region increases, reaching a maximum value (between $1200-1400 \mathrm{~nm}$ ) beyond which it starts to decrease. Nonetheless, for a scenario dominated by field-assisted dark-current generation both the peak SPQE and the FWHM (full-width-athalf-maximum) of each curve increase as the width of the multiplication region increases (dashed lines). The existence of an optimal peak SPQE at room temperature is a result of the competing effects of the field-and temperature-assisted generation of dark carriers.

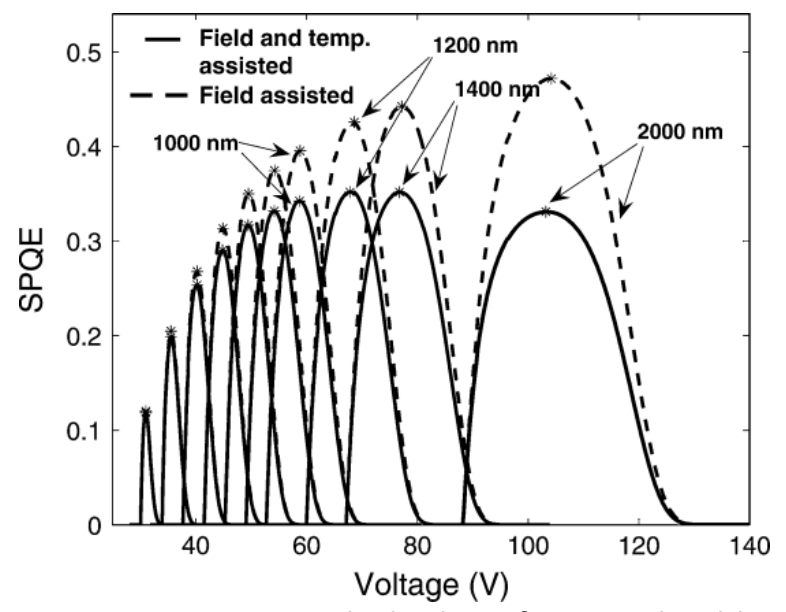

Fig. 5. SPQE versus applied voltage for several widths of the multiplication region. The maximum achievable value of the SPQE curve is determined by the quantum efficiency $\eta$, which in this case is 0.5 . 


\section{B. InAIAs-InP Heterojunction Multiplication Regions}

Thin heterojunction multiplication regions have proven to be beneficial in reducing the excess noise factor due to the strong effect of the dead space in devices with thin multiplication region [23]-[24][25]. However, their desirable characteristics decrease in devices with thick multiplication regions due to the reduced importance of dead space in these devices. Additionally, in an earlier theoretical work [12] we had shown that the fractional width of the $\mathrm{In}_{0.52} \mathrm{Al}_{0.48} \mathrm{As}$ layer in an $\mathrm{In}_{0.52} \mathrm{Al}_{0.48} \mathrm{As}$-InP heterojunction multiplication region can be optimized to attain a maximum SPQE that is greater than that offered by a homojunction InP multiplication region.

(The fractional width of the $\operatorname{In}_{0.52} \mathrm{Al}_{0.48} \mathrm{As}$ layer in an $\ln _{0.52} \mathrm{Al}_{0.48} \mathrm{As}-\mathrm{InP}$ heterojunction multiplication region is defined as the ratio between the width of the $\ln _{0.52} \mathrm{Al}_{0.48} \mathrm{As}$ energy buildup layer to the total width of the heterojunction multiplication region comprising the $\ln _{0.52} \mathrm{Al}_{0.48} \mathrm{As}$ and $\mathrm{InP}$ layers.) It was also shown that this effect became more pronounced in thin multiplication regions as a result of the increased significance of dead space. Therefore, it would be of interest to further investigate the performance of SPADs with heterojunction multiplication regions. It should be pointed out that in our simulations of the InAIAs-InP heterojunction we have used the values of the parameters $\tau_{\text {eff }}, N_{T}$ and a corresponding to $\ln P$ throughout the entire multiplication region due to lack of precise knowledge of the values of these parameters for InAlAs.

Fig. 6 shows the DCR as a function of the normalized excess voltage for four different widths of the multiplication region. By comparing Fig. 6 with Fig. 3, we observe that the curves show a similar trend in the DCR as the width of the multiplication region is varied. Similarly to the case of a homojunction multiplication region and in accord with our understanding of the dominance of field-assisted effects over GR effects in high-fields, the change in the $D C R$, as the role of GR is varied (for a certain width of the multiplication region), is only noticeable in thicker multiplication regions.

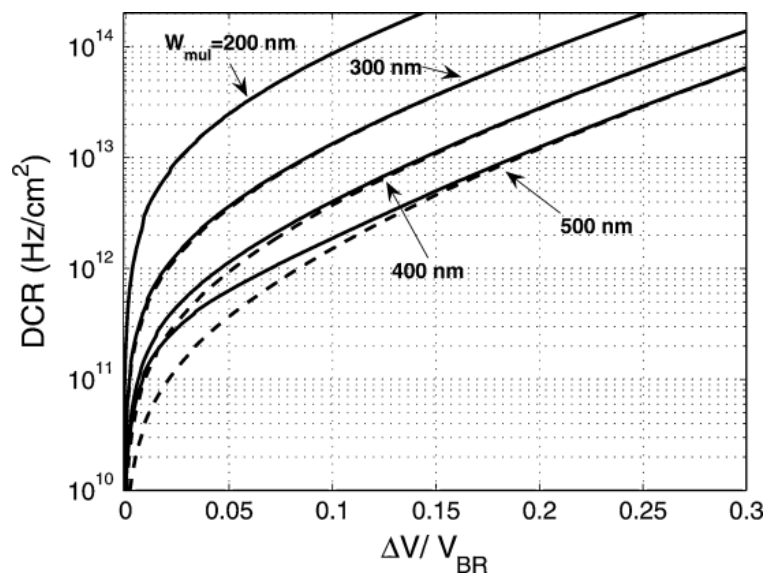

Fig. 6. DCR versus normalized excess voltage for $200 \mathrm{~nm}, 300 \mathrm{~nm}, 400 \mathrm{~nm}$, and $500 \mathrm{~nm}$ multiplication region widths. Dashed lines correspond to the case when GR dark carriers are absent and solid lines correspond to the case when both fieldassisted and GR dark carriers are present.

The SPQE curves, on the other hand, give us a slightly more informative account of things. As a function of the applied voltage, the SPQE exhibits a different behavior in the cases of a homojunction and heterojunction multiplication regions. Fig. 7 shows the calculated SPQE versus the applied voltage for the homojunction and heterojunction multiplication regions for several widths of the multiplication region. For a given width of the multiplication region, the calculated SPQE of the heterojunction multiplication region is higher than that for the homojunction case. Moreover, this enhancement in the SQPE, as we move from a homojunction to a heterojunction, becomes more pronounced as the width of the multiplication region is reduced. This is attributed to the fact that for a given width of the multiplication region, the electric field required to achieve a certain breakdown probability is smaller in the heterojunction multiplication-region case than that in a 
homojunction multiplication-region case [12], which, in turn, results in a reduction in the number of dark carriers generated through field-assisted mechanisms. The improvement in breakdown characteristics in properly designed heterojunction multiplication layers is a result of the so-called initial-energy effect, which takes advantage of injecting "hot" carriers from a high bandgap layer (InAlAs in our case) of the multiplication region to the lower bandgap layer (InP) [12], [19]. It is to be noted, however, that this conclusion does not take into account the possibility of an increase in hole trapping in a heterojunction multiplication region, which may aggravate after-pulsing.

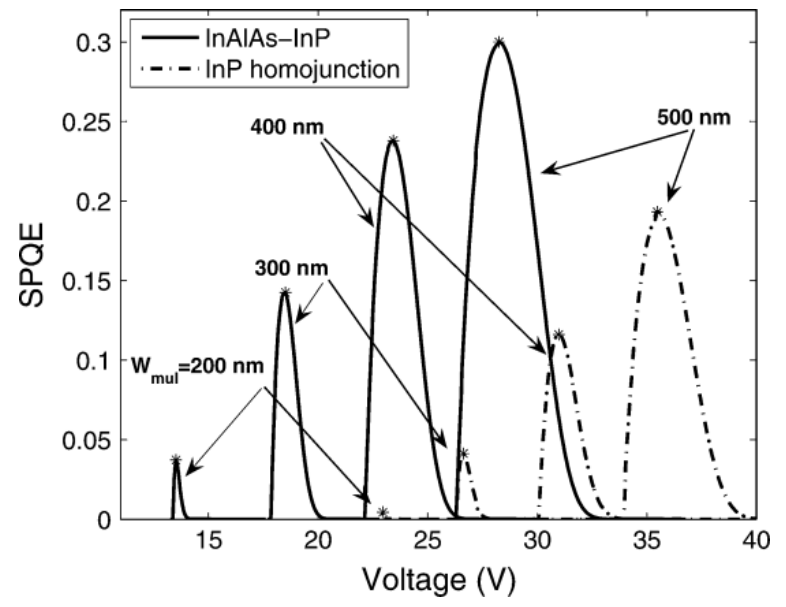

Fig. 7. SPQE versus applied voltage for InP homojunction and InAIAs-InP heterojunction multiplication region.

The effect of afterpulsing was neglected in our study primarily due to the lack of precise knowledge of the dependence of the carrier-release time on the field. For example, increasing the multiplication-region width results in an increase in the number of traps while lowering the field results in an increase in the carrier-release time. Both of these factors may lead to a stronger afterpulsing.

As a final remark we note that electron-phonon coupling (not included in our study) gives rise to thermally assisted tunneling [26]-[27][28]. In phonon-assisted tunneling the electron absorbs thermal energy from the lattice and then tunnels through the barrier at a higher energy [27]. This effect increases the number of dark carriers created through tunneling. As this effect has a field dependence that is of similar form to that for bandto-band tunneling, we do not expect it to impact the trends predicted by our study. For example, our calculations (graphs not included) show that doubling the number of dark carriers created through tunneling in Fig. 5 will not alter the predicted trend in the peaks of the SPQE.

\section{SECTION IV. Conclusion}

Our theoretical study shows that the thickness of the multiplication region plays a different role in the performance of a SPAD depending upon what mechanism of dark-carrier generation is dominant. At low temperatures, for which field-assisted mechanisms are dominant, an increment in the thickness of the multiplication region will result in an improved PDE versus DCR characteristics. The same behavior is seen in the SPQE curve at low temperatures. At room temperatures, on the other hand, the PDE versus DCR characteristics show a weaker performance as the width of the multiplication region is increased. However, the SPQE curves show a maximum achievable peak SPQE at an optimal overbias and an optimal multiplication-region width. It is important to note that the behavior of an APD as a function of the multiplication-region width in the linear mode, where excess noise factor decreases as the multiplication-region width is decreased, is characteristically different from that of a SPAD. 


\section{References}

1. C. P. Morath, K. Vaccaro, W. R. Clark, W. A. Teynor, M. A. Roland, W. Bailey, "Performnace characterization of an InGaAs/InP single photon avalanche diode", Proc. 49th Annu. Meeting SPIE: Applications of Digital Image Processing XXVII, 2004.

2. A. Biswas, W. H. Farr, "Detectors for ground-based reception of laser communications from Mars", Proc. Lasers and Electro-Optics Society (LEOS) 17th Annu. Meeting, vol. 1, pp. 74-75, 2004.

3. A. Spinelli, L. M. Davis, H. Dautet, "Actively quenched single-photon avalanche diode for high repetition rate time-gated photon counting", Rev. Sci. Instrum., vol. 67, pp. 55-61, 1996.

4. D. Stucki, G. Ribordy, H. Z. A. Stefanov, J. G. Rarity, "Photon counting for quantum key distribution with Peltier cooled InGaAs/InP APD's", J. Modern Optics, vol. 48, pp. 1967-1981, 2001.

5. M. B. Nasr, B. E. A. Saleh, A. V. Sergienko, M. C. Teich, "Dispersion-cancelled and dispersion-sensitive quantum optical coherence tomography", Opt. Express, vol. 12, pp. 1353-1362, 2004.

6. H. Zbinden, H. Bechmann-Pasquanucci, N. Gisin, G. Ribordy, "Quantum cryptography", Appl. Phys. B., vol. 67, pp. 743-748, 1998.

7. J. M. Merolla, Y. Mazurenko, J. P. Goedgebuer, L. Duraffourg, H. Porte, W. T. Rhodes, "Quantum cryptographic device using single-photon phase modulation", Phys. Rev. A, vol. 60, pp. 1899-1905, 1999.

8. Y. Kang, H. X. Lu, Y. H. Lo, D. S. Bethune, W. P. Risk, "Dark count probability and quantum efficiency of avalanche photodiodes for single-photon detection", Appl. Phys. Lett., vol. 83, pp. 2955-2957, 2003.

9. K. A. Mcintosh, J. P. Donnelly, D. C. Oakley, A. Napoleone, S. D. Calawa, L. J. Mahoney, K. M. Molvar, E. K. Duerr, S. H. Groves, D. C. Shaver, "InGaAsP/InP avalanche photodiodes for photon counting at 1.06 $\mu \mathrm{m} "$, Appl. Phys. Lett., vol. 81, pp. 2505-2507, 2002.

10. J. P. Donnelly, E. K. Duerr, K. A. Mcintosh, E. A. Dauler, D. C. Oakley, S. H. Groves, C. J. Vineis, L. J. Mahoney, K. M. Molvar, P. I. Hopman, K. E. Jensen, G. M. Smith, S. Verghese, D. C. Shaver, "Design considerations for 1.06- $\mu \mathrm{m}$ InGaAsP-InP Geiger-mode avalanche photodiodes", IEEE J. Quantum Electron., vol. 42, no. 8, pp. 797-809, 2006.

11. M. M. Hayat, U. Sakoğlu, O.-H. Kwon, S. Wang, J. C. Campbell, B. E. A. Saleh, M. C. Teich, "Breakdown probabilities for thin heterostructure avalanche photodiodes", IEEE J. Quantum Electron., vol. 39, pp. 179-185, 2003.

12. D. A. Ramirez, M. M. Hayat, G. Karve, J. C. Campbell, S. N. Torres, B. E. A. Saleh, M. C. Teich, "Generalized breakdown probabilities and detection efficiencies for single-photon avalanche photodiodes", IEEE J. Quantum Electron., vol. 42, no. 2, pp. 137-145, 2006.

13. S. R. Forrest, R. F. Leheny, R. E. Nahony, M. A. Pollack, "In 0.53Ga0.47As photodiodes with dark current limited by generation-recombination and tunneling", Appl. Phys. Lett., vol. 37, no. 3, pp. 322-325, 1980.

14. G. Karve, S. Wang, F. Ma, X. Li, J. C. Campbell, "Origin of dark counts in In 0.53Ga0.47As/In0.52Al0.4As avalanche photodiodes operated in Geiger mode", Appl. Phys. Lett., vol. 86, 2005.

15. S. Cova, M. Ghioni, A. Lotito, I. Rech, F. Zappa, "Evolution and prospects for single-photon avalanche diodes and quenching circuits", J. Modern Opt., vol. 51, pp. 1267-1288, 2004.

16. M. A. Saleh, M. M. Hayat, P. Sotirelis, A. L. Holmes, J. C. Campbell, B. E. A. Saleh, M. C. Teich, "Impactionization and noise characteristics of thin III-V avalanche photodiodes", IEEE Trans. Electron Devices, vol. 48, pp. 2722-2731, 2001.

17. J. S. Ng, C. H. Tan, J. P. David, G. Hill, G. J. Rees, "Field dependence of impact ionization coefficients in In 0.5Ga0.47As", IEEE Trans. Electron Devices, vol. 50, no. 4, pp. 901-905, Apr. 2003.

18. F. Osaka, T. Mikawa, T. Kaneda, "Electron and hole ionization coefficients in (100) oriented Ga0.33In0.67As0.70P0.30", Appl. Phys. Lett, vol. 45, no. 3, pp. 292-293, 1984. 
19. M. M. Hayat, O.-H. Kwon, S. Wang, J. C. Campbell, B. E. A. Saleh, M. C. Teich, "Boundary effects on multiplication noise in thin heterostructure avalanche photodiodes: Theory and experiment", IEEE Trans. Electron Devices, vol. 49, no. 12, pp. 2114-2123, 2002.

20. S. L. Chuang, Physics of Optoelectronic Devices, New York:Wiley, 1995.

21. Y. Rosenwaks, Y. Shapira, D. Huppert, "Picosecond time-resolved luminescence studies of surface and bulk recombination processes in InP", Phys. Rev. B., vol. 45, no. 16, pp. 9108-9119, 1992.

22. Y. L. Goh, D. J. Massey, A. R. J. Marshall, J. S. Ng, C. H. Tan, W. K. Ng, G. J. Rees, M. Hopkinson, J. P. R. David, S. K. Jones, "Avalanche multiplication in InAIAs", IEEE Trans. Electron Devices, vol. 54, no. 1, pp. 11-16, 2007.

23. B. E. A. Saleh, M. M. Hayat, M. C. Teich, "Effect of dead space on the excess noise factor and time response of avalanche photodiodes", IEEE Trans. Electron Devices, vol. 37, pp. 1976-1984, 1990.

24. M. M. Hayat, W. L. Sargeant, B. E. A. Saleh, "Effect of dead space on gain and noise in Si and GaAs avalanche photodiodes", IEEE J. Quantum Electron., vol. 28, pp. 1360-1365, 1992.

25. O.-H. Kwon, M. M. Hayat, S. Wang, J. C. Campbell, A. Holmes, B. E. A. Saleh, M. C. Teich, "Optimal excessnoise reduction in thin heterojunction Al0.6Ga0.4As/GaAs avalanche photodiodes", J. Quantum Electron., vol. 39, pp. 1287-1296, 2003.

26. G. Vincent, A. Chantre, D. Bois, "Electric field effect on the thermal emission of traps in semiconductor junctions", J. Appl. Phys., vol. 50, pp. 5484-5487, 1979.

27. P. A. Martin, B. G. Streetman, K. Hess, "Electric field enhanced emission from non-Coulombic traps in semiconductors", J. Appl. Phys., vol. 52, pp. 7409-7415, 1981.

28. G. A. M. Hurkx, D. B. M. Klaassen, M. P. G. Knuvers, "A new recombination model for device simulation including tunneling", IEEE Trans. Electron Devices, vol. 39, pp. 331-338, 1992. 